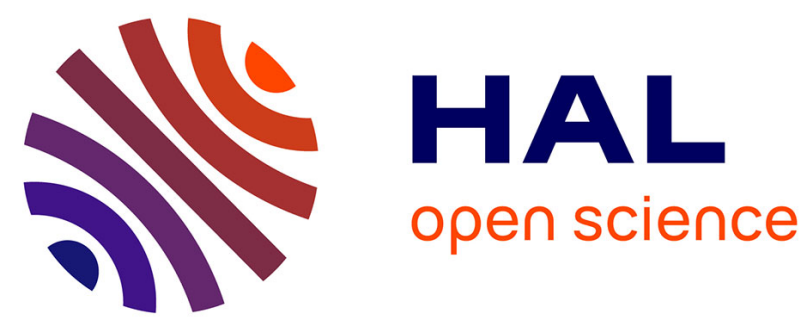

\title{
Les potins " à la tête diabolique "
}

Florence Barthélémy

\section{To cite this version:}

Florence Barthélémy. Les potins " à la tête diabolique ". Gallia - Archéologie de la France antique, 1995, Les potins gaulois, 52, pp.27-36. 10.3406/galia.1995.3118 . hal-01911263

\section{HAL Id: hal-01911263 https://hal.science/hal-01911263}

Submitted on 7 Feb 2020

HAL is a multi-disciplinary open access archive for the deposit and dissemination of scientific research documents, whether they are published or not. The documents may come from teaching and research institutions in France or abroad, or from public or private research centers.
L'archive ouverte pluridisciplinaire $\mathbf{H A L}$, est destinée au dépôt et à la diffusion de documents scientifiques de niveau recherche, publiés ou non, émanant des établissements d'enseignement et de recherche français ou étrangers, des laboratoires publics ou privés.

\section{(이) $\$$}

Distributed under a Creative Commons Attribution - NonCommercial - NoDerivatives 44.0 


\title{
2.3. Les potins « à la tête diabolique»
}

\section{Florence Barthélémy}

\begin{abstract}
Résumé. Les potins dits "à la tête diabolique » ont eu une large répartition sur toute la France. Cépendant, on attribue généralement leur fabrication aux Turones, car les plus fortes concentrations de ces potins se trouvent dans la région de Tours et d'Amboise. La première classification lypologique proposée par J.-B. Colbert de Beaulieu distinguait deux classes. L'étude du matériel trouvé sur le sanctuaire d'Allonnes (Sarthe) montre que la classe II de Colbert de Beaulieu recouvre des variations suffisamment évidentes pour que l'on puisse la diviser en plusieurs classes (nos classes II à VIII). Notre proposition de classification distingue parfois des divisions à l'intérieur d'une même classe, lorsque des différences typologiques ou métriques existent mais restent moins perceptibles à l'œil que les éléments de ressemblance. La validité de celte typologie a ensuite élé testée sur le trésor de Fondettes (Indre-et-Loire).
\end{abstract}

\begin{abstract}
Zusammenfassung. Die Potinmünzen "à la tête diabolique " waren reichlich über ganz Frankreich verbreitet. Jedoch wird generell deren Herstellung den Turonen zugeschrieben, denn die Höchstkonzentrationen dieser Potinmünzen finden sich im Gebiet von Tours und Amboise. Die erste, ron J.-B. Colbert de Beaulieu vorgeschlagene typologische Eingliederung, unterschied zwei Klassen. Die Bearbeilung des Fundmaterials des Sanktuars von Allonnes (Sarthe) zeigt, das Colbert de Beaulieu 's Klasse II genügend deutliche Varianten aufweisl, und sich eine Aufleilung in weiteren Klassen aufdrängt (CF. unsere Klassen II bis VIII). Unser Klassifizierungsvorschlag unterscheidet weitere Unterteilungen innerhalb einer Klasse, dort nämlich, wo typologische oder metrische Unterschiede wohl zu finden sind, jedoch aber weniger auffälig, als die Elemente der Ähnlichkeit sind. Die Berechligung dieser typologie wurde später am Fondeltes-Fund (Indre-et-Loire) erprobt.
\end{abstract}

Les potins dits " à la tête diabolique " ont eu une large répartition sur toute la France.

J.-B. Colbert de Beaulieu a présenté la première et seule classification des potins " à la tête diabolique " dans son article de la Revue Belge de Numismatique (RBN) paru en 1970. Il distingue deux classes : la classe I est du type BN 5684, la classe II est du type BN 5675. Si la classe I est bien définie, la classe II, moins précise, est constituée de types dont l'iconographie très diversifiée ne permet pas une typologie très rigoureuse. Pour sa description, il cite notamment E. Lambert : "Les types sont ordinairement une tête très grossièrement dessinée, le contour du nez indiqué par une grosse ligne, quelquefois ponctuée au bout, un globule ou un trou pour former l'œil, un ou deux globules aplatis pour représenter les lèvres, une proéminence sphérique complète le reste de la tête."

Il était temps de reprendre l'étude de ces objets monétiformes. Nous disposons pour cela de neuf cent cinquante-trois pièces : deux cent vingt-quatre proviennent du sanctuaire d'Allonnes, cent neuf du lot du Cabinet des médailles de la Bibliothèque nationale de France (provenance inconnue), quatre cent quatre-vingt-quatorze sont issues du trésor de Fondettes, cinquante-six viennent des fouilles d'Amboise, soixante-dix émanent de sources diverses dont les provenances sont inconnues.
TYPOLOGIE (FIG. 10)

Ellc comporte huit classes dont certaines peuvent se subdiviser en plusieurs variantes.

\section{Classe I : type BN 5684}

Le droit présente le profil à gauche d'une tête humaine. Cette classe est divisée en deux : la classe Ia a les cheveux constitués de trois mèches; la classe Ib possède les cheveux formés par deux ou trois $\mathrm{S}$ affrontés. L'ensemble de la pièce est limité par un bourrelet circulaire. Géométriquement, la tête a la forme d'un losange. Cette remarque est intéressante car elle permet de reconnaître cette classe lorsque la pièce est très usée.

Le revers est identique pour les deux sous-classes. Il représente un taureau cornu tourné à gauche. Il est bien défini. I a patte avant est repliée sous l'abdomen, la patte arrière longe le bourrelet circulaire. La queue est relevée en forme de $S$ au-dessus du corps de l'animal. Une petite croix de saint André se remarque au-dessus de l'encolure lorsque la monnaie n'est pas trop érodée.

Le module de cette série est assez petit. Il varie de $12 \mathrm{~mm}$ à $16 \mathrm{~mm}$ (on obtient une moyenne de $14 \mathrm{~mm}$ ). Le poids oscille entre $1,38 \mathrm{~g}$ et $3,51 \mathrm{~g}$ (la moyenne est de $2,01 \mathrm{~g}$ ). 
Typologie des potins dits «à tête diabolique»
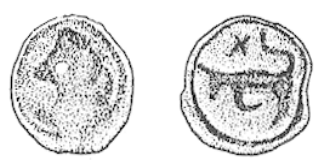

Classe la: BN 5684
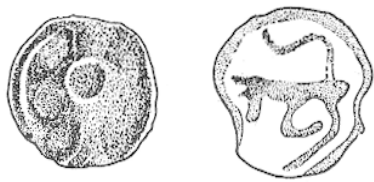

Classe II: BN 5675
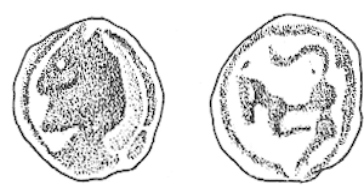

Classe IV: BN 5669
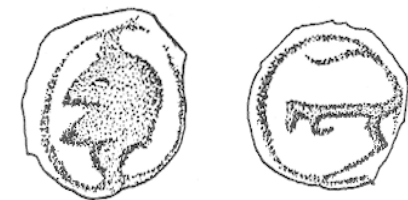

Classe VI: BN 5673
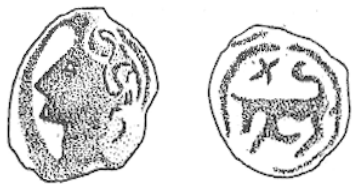

Classe Ib
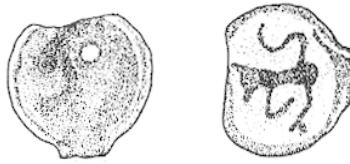

Classe III: BN 5687

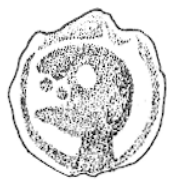

Classe V: BN 5656

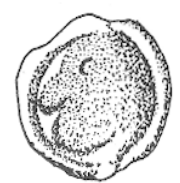

Classe VII: BN 5669
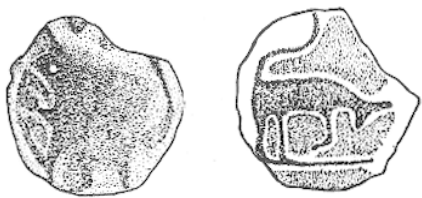

Classe VIII

Fig. 10. Typologie des potins " à la lête diabolique ". Dessins réalisés d'après les polins d'Allones par J.-F. Enault, UMR 126-6 du CNRS.

\section{Classe II : type BN 5675}

Au droit, une tête humaine à gauche est très schématisée. L.e nez est droit, le menton pointu. L'oeil est rendu par une profonde cavité très importante et régulière sur toutes les pièces de ce type. Le contour de la tête est donné uniquement par son relief. On remarque que le haut du crâne se rétrécit et se prolonge jusqu'au listel en rejoignant le jet de coulée. La tête peut être plus ou moins importante, c'est-à-dire qu'elle remplit plus ou moins la face de la pièce.

Au revers, le quadrupède est à gauche. La queue est recourbée au-dessus du dos. La patte avant est repliée sous son abdomen. La patte arrière longe plus ou moins le bourrelet. Quelquefois, cette dernière est fourchue à son extrémité. Sur l'ensemble de cette classe, la représentation bovine présente quelques différences iconographiques telles que la position de la queue qui est plus ou moins en forme de $S$, la patte arrière qui est plus ou moins repliée...., mais ces éléments sont trop peu visibles et remarquables pour rendre nécessaire la création d'autres classes ou sous-classes complémentaires. On doit $\mathrm{y}$ voir simplement un rendu différent de la gravure initiale, ces pièces ne sortant pas de la même alvéole du moule.

En revanche, les très grandes différences de modules autorisent la division de cette classe en trois groupes, ce qui permet un repérage plus précis car la classe II est très abondante. On notera :

- classe IIa : diamètre inférieur à $15 \mathrm{~mm}$;

- classe IIb : diamètre compris entre $15 \mathrm{~mm}$ et $17 \mathrm{~mm}$;

- classe IIc : diamètre supérieur ou égal à $17 \mathrm{~mm}$.

\section{Classe III : type BN 5687}

Au droit, la tête est identique en tous points à celle de la classe II.

Le revers représente un petit taureau de bonne facture. La patte arrière s'étire vers l'avant en longeant le bourrelet circulaire de la pièce. La queue forme un $\mathrm{S}$ au-dessus du dos de l'animal. La particularité du graphisme du quadrupède est qu'il est "bouleté " : c'est-à-dire que le crâne, l'épaule, la croupe sont mis en relief par de petites masses hémisphériques. L'ensemble donne l'impression que le corps est plus en relief que les membres (pattes et queue).

Le module varie de $12,6 \mathrm{~mm}$ à $16,6 \mathrm{~mm}$ et la moyenne obtenue est de $14,6 \mathrm{~mm}$. Quant au poids, il est compris entre $1,31 \mathrm{~g}$ et $5,52 \mathrm{~g}$ (la moyenne est de $2,22 \mathrm{~g}$ ).

\section{Classe IV : type BN 5669}

Nous pourrions l'appeler « le potin à la tête atrophiée ". En effet, comparativement à celle de la classe II, la têtce est très déformée. Si la partie arrière du crâne est pratiquement identique, ainsi que la position de l'oeil que l'on note néanmoins de forme plus réduite, toute la face diffère : le nez se devine à peine et est situé très près de l'œil. Ia mandibule, excessivement réduite, rejoint directement le nez. Le maxillaire sous l'œil est proéminent.

Le revers est identique à celui de la classe III. C'est un petit taureau bouletè. Le diamètre est compris entre 
$14,10 \mathrm{~mm}$ et $17 \mathrm{~mm}$. On obtient une moyenne de $15,50 \mathrm{~mm}$. Le poids diffère de $1,68 \mathrm{~g}$ à $2,66 \mathrm{~g}$. La moyenne est de $2,10 \mathrm{~g}$.

Cette classe, si ressemblante à la classe précédente, en serait-elle une dégénérescence? Serait-elle une mauvaise série de la classe III? On pourrait penser que c'est le résultat d'un défaut de gravure ou d'incident de fabrication. Cependant, il en existe de nombreux exemplaires.

\section{Classe V : type BN 5656}

Le droit montre le profil gauche d'un visage humain. L'occupation de la tête est assez imposante et est bien démarquée par rapport au listel. Le crâne est très arrondi à l'arrière; l'œil bien prononcé est cependant moins profond et de module inférieur à celui de la classe II. Le menton et le nez sont proéminents, plutôt pointus. La caractéristique essentielle de cette classe est la présence de lèvres : elles sont symbolisées par un ou deux globules aplatis, situés entre le nez et le menton, placés verticalement ou parfois obliquement.

Au revers, le taureau à gauche est assez massif. La tête et le corps sont bien proportionnés (on trouve une grande similitude avec celui de la classe VII). La queue est en $\mathrm{S}$ au-dessus du dos. La patte arrière est quelquefois fourchue à son extrémité ou bien forme une ligne de sol.

En ce qui concerne les mesures, le diamètre est compris entre $15 \mathrm{~mm}$ et $16,9 \mathrm{~mm}$ (la moyenne est de $15,92 \mathrm{~mm}$ ) et le poids oscille entre $2,22 \mathrm{~g}$ et $3,75 \mathrm{~g}$ (la moyenne obtenue est de $2,98 \mathrm{~g}$ ).

\section{Classe VI : type BN 5673}

Au droit, le profil de la tête humaine est tourné à gauche. Elle est semblable à celle de la classe II décrite précédemment.

Au revers, l'image du taureau est très simplifièe. Le corps de l'animal se réduit à une simple ligne horizontale. La tête de l'animal est quelquefois visible, mais l'arrière forme un cylindre continu qui se perd dans le bourrelet circulaire. Cette ligne qui forme le corps est plus en relief que les pattes et la queue de l'animal. La patte avant est repliée sous l'abdomen; celle de l'arrière, courbée vers l'avant, rejoint le listel de la pièce environ à la moitié du corps. La queue forme un S au-dessus du dos du taureau.

Le diamètre de cette pièce est remarquable. Il est compris entre $14,8 \mathrm{~mm}$ et $18,6 \mathrm{~mm}$. La moyenne obtenue est de $17 \mathrm{~mm}$. Le poids, qui n'est pas un élément caractéristique, varie de $1,68 \mathrm{~g}$ à $3,15 \mathrm{~g}$. I a moyenne est de $2,38 \mathrm{~g}$.

\section{Classe VII : type BN 5669}

Au droit, la tête humaine remplit tout l'espace. L'œil, de petit diamètre, se caractérise par un trou circulaire, profond, en haut à gauche de la tête. Les contours de la face sont peu précis. Le relief de la gravure est donné uniquement par le creux périphérique existant entre le bourrelet de la pièce et le modèle.

Au revers, le taureau est à gauche. C'est une représentation massive de l'animal (très voisine du graphisme de la classe V). La patte arrière sert quelquefois de ligne de sol. I a queue est une sinusoïde plus ou moins prononcée au-dessus du dos.

I.e diamètre le moins important est de $15 \mathrm{~mm}$, le plus grand est de $19 \mathrm{~mm}$. Ceci donne une moyenne de $16,35 \mathrm{~mm}$. Le poids varie de $1,89 \mathrm{~g}$ à $3,51 \mathrm{~g}$. La moyenne obtenue est de $2,95 \mathrm{~g}$.

\section{Classe VIII}

Le droit est identique à celui de la classe VII décrite cidessus.

$\mathrm{Au}$ revers, on trouve les mêmes caractéristiques que pour le taureau de la classe VII ; cependant, il est en position de charge tourné à droite contrairement aux autres classes et possède deux pattes arrière. Que peut-on supposer de la position du taureau? Serait-ce la spécialité d'un atelier? Actuellement, nous ne pouvons pas expliquer cette originalité. Il faut cependant remarquer que cette série est absente du trésor de Fondettes.

Le diamètre varie de $16 \mathrm{~mm}$ à $20,2 \mathrm{~mm}$. On obtient une moyenne de $17,4 \mathrm{~mm}$. En ce qui concerne la fluctuation des poids, le plus petit est de $2,54 \mathrm{~g}$ et le plus lourd de 7,08 g. La moyenne réalisée est de 3,70 g.

Cette typologie a été réalisée dans un premier temps à partir des monnaies d'Allonnes et vérifiée sur le lot de la Bibliothèque nationale de France puis sur le trésor de Fondettes. Cette classification est cohérente et trouve sa justification dans tous les cas rencontrés. Les huit classes créées portent des numéros qui ne correspondent pas à un ordre chronologique mais à une première tentative d'évolution typologique et métrologique. I es renseignements fournis par les découvertes actuelles ne permettent pas de préciser les liens les unissant les uns aux autres. 


\section{RÉPARTITION GÉOGRAPHIQUE (FIG. 11)}

L'inventaire détaillé des lieux de découvertes des potins «à la tête diabolique » est présenté ici dans l'ordre alphabétique des départements. Chaque nom de site est suivi du nombre de pièces connues ainsi que des références bibliographiques où sont mentionnés ces renseignements.

AISNE

1. Condé-sur-Aisne : quantité non répertoriée

Blanchet, 1905, p. 251, fig. 116; GiARD J.-B., Le pèlerinage gallo-romain de Condé-sur-Aisne et ses monnaies, $R N, \mathrm{X}, 1968$, p. 102 ; Mitard, 1967, p. 88.

2. Pommiers : 14

FOUCART J., Mémoires des Sociétés d'Histoire de l'Aisne, 1973.

3. Villeneuve-Saint-Germain : 1

DEBORD J., Monnaies gauloises de Villeneuve-SaintGermain, Cahier Archéologique de Picardie, 5, 1978, p. 111; DEBORD J., Premier bilan de huit années de fouilles à Villeneuve-Saint-Germain, Revue Archéologique de Picardie, 1973-1980, p. 252 (numéro spécial Vallée de l'Aisne).

\section{ALLIER}

\section{Néris-les-Bains : 1}

Colbert de Beaulieu, 1970, p. 109.

\section{BAS-RHIN}

\section{Strasbourg : 2}

Blanchet, 1905, p. 511.

\section{BOUCHES-DU-RHÔNE}

\section{Lançon-Provence : 1}

VIAN P.-C., La circulation des monnaies gauloises dans la région du Vaucluse, OGAM, VI, 1954, fasc. 5, p. 249254

\section{Saint-Rémy-de-Provence : 3}

Rolland H., Numismatique de Glanum, Cahiers d'Histoire et d'Archéologie, 12, 1932, p. 7, n²6.

\section{Calvados}

8. Caen : quantité non répertoriée

Mitard, 1967, p. 87.

\section{CHARENTE}

9. Chassenon : quantité non répertoriée Blanchet, 1905, p. 251.

\section{ChARENTE-MARITIME}

\section{Muron : 4}

MAINJONET M., Monnaies gauloises récoltées à Muron, Revue de la Saintonge et de l'Aunis, I, 1975, p. 49-56.

\section{CHER}

\section{Bourges : 4}

Blanchet, 1905, p. 507.

12. Dun-sur-Auron : 1

Colbert de Beaulieu, 1970, p. 107.

13. Neuvy-sur-Barangeon : 18

Mitard, 1967, p. 88.

14. Saint-Amand-Montrond : 1

Colbert de Beaulieu, 1970, p. 109.

15. Saint-Satur : 1

COI.BERT DE BEAULIEU J.-B., À l'occasion d'une trouvaille inédite des environs de Fougères : la monnaie de potin « à la tête diabolique ", Annales de Bretagne, 1961, p. 50, note 19 .

16. Senneçay : quantité non répertoriée

Blanchet, 1905, p. 251, n 1 .

\section{CÔTE-D'OR}

17. Alise-Sainte-Reine : quantité non répertoriée

Colbert de Beaulieu, 1970, p. 105.

18. Flavignerot : quantité non répertoriée.

Colbert de Beaulieu, 1970, p. 107.

19. Mâlain : 1

FISCHER B., Les monnaies gauloises de Mâlain, $R A E$, 3-4, 1985, p. 229-236.

20. Nuits-Saint-Georges : 1

LE DANTEC J.-P., Monnaies gauloises recueillies sur le site des Bolards : le manuscrit de Louis Roux, RAE, 23, 1972, p. 45-47.

\section{CÔTES-D'ARMOR}

21. Corseul : 1

CHICHÉ B., Récente découverte de monnaies gauloises à Corseul, Cahiers Numismatiques, Bulletin de la SENA, 1970, 7, p. 114.

22. Lannion : quantité non répertoriée

Mitard, 1967, p. 87.

\section{EURE-ET-LOIR}

23. Chartres : 4

Fischer, 1986, p. 102.

24. Châteaudun : 8

Fischer, 1986, p. 102. 


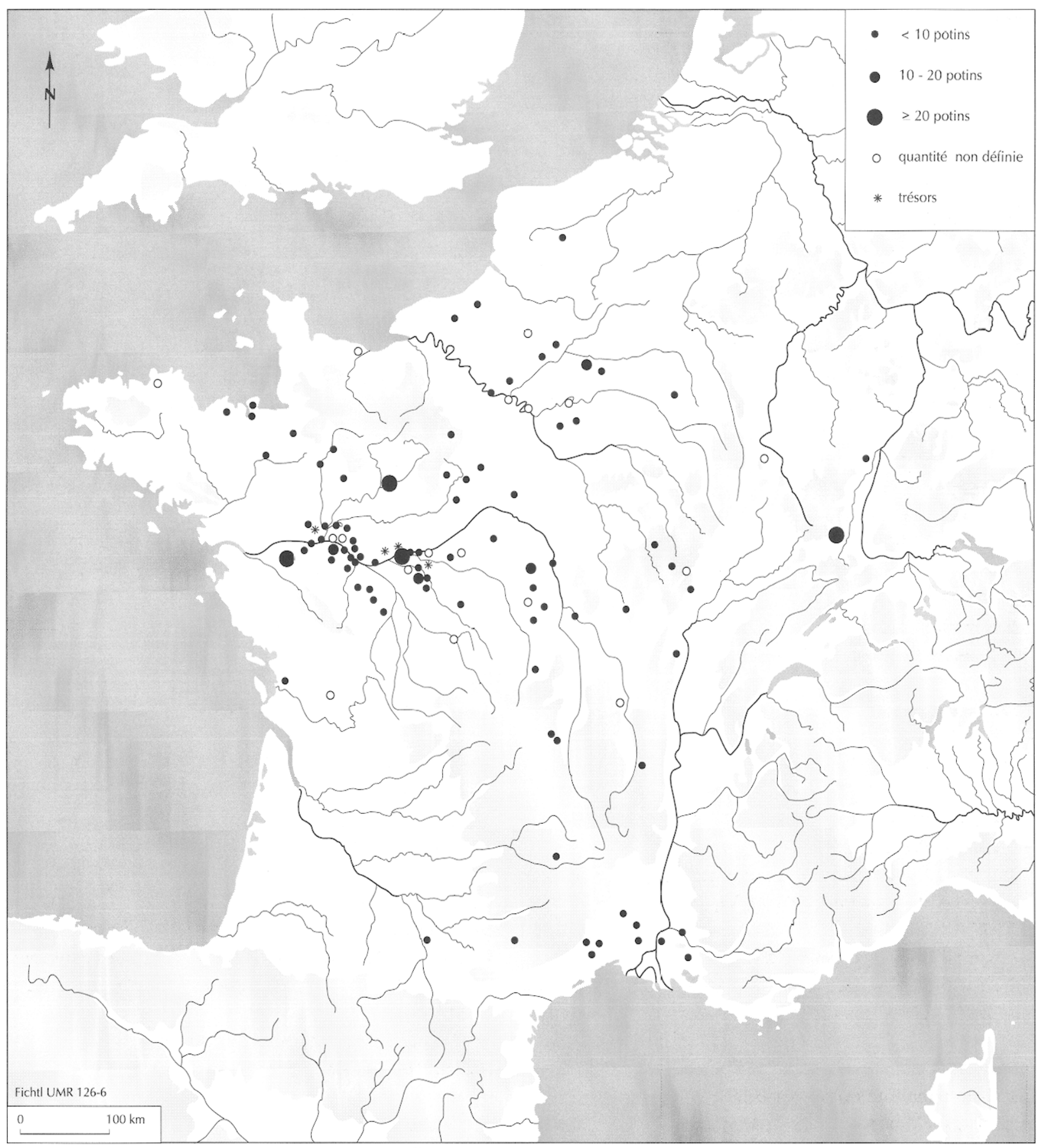

Fig. 11. Carte de répartition des potins "à la tête diabolique». 
25. Mérouville : 1

Présent au Musée de Saint-Germain-en-Laye, classé 1376 (4476).

26. Puerthe : 2

Fischer, 1986, p. 102.

GARD

27. Alès : 1

Vian, 1966, 3, p. 260-263.

28. Brignon : 2

Vian, 1966, 3, p. 260-263.

29. Saint-Gilles : 1

RICHARD J.-C., Les monnaies du domaine d'Espeyran, RAN, 11, 1978, p. 101-104.

\section{HAUTE-GARONNE}

30. Vieille-Toulouse : 3

LABROUSSE M., Monnaies gauloises de la collection Azéma au musée du Vieux-Toulouse. IV : Monnaies du centre de la Gaule, Pallas, IX, 2, 1960, p. 213-214.

HAUT-RHIN

31. Mulhouse : 20

Mitard, 1967, p. 87.

\section{HÉRAULT}

32. Castelnau-le-Lez : 1

Depeyrot G., AlbaGnaC L., État des découvertes et de la circulation monétaire dans la région de Montpellier, Numisma, 28, 1978, p. 241-306.

33. Lattes : 1

Majurel R., Arnal J., Prades H., Deux nouveaux trésors de Lattes, OGAM, 19, 1967, p. 397-433.

34. Murviel-lès-Montpellier : 5

RICHARD J.-C., SOYRIS P., Notes de numismatique narbonnaise. III : Les monnaies de l'oppidum du Castellas à Murviel-lès-Montpellier (1950-1975), RAN, 9, 1976, p. 219-245.

\section{IL.LE-ET-VILAINE}

35. Fougères : 34

Colbert de Beaulieu, 1961, 1, p. 47.

36. Rennes : 1

COLBERT DE BEAUlIEU J.-B., Monnaies gauloises trouvées à Rennes dans la Vilaine, Annales de Bretagne, LXVII, 1, 1960 , p. 82-83, fig. B10.

37. Saint-Malo : 3

Colbert de Beaulieu, 1961, 1, p. 45.
38. Saint-Servan : 1

$C A G 35,288$.

INDRE

39. Levroux : 7

Brunaux, Gruel, 1987, p. 138-149.

40. Saint-Marcel : quantité non répertoriée

Gallia, 42, 1984, p. 318-319.

\section{INDRE-ET-LOIRE}

41. Amboise : quantité non définie

CARTIER E., Supplément aux dix lettres sur l'histoire monétaire de France, $R N, 1842$, p. 419-445; ColIN A., La chronologie des oppida en France non méditerranéenne, thèse de Doctorat (O. Buchsenschutz. dir.), université de Paris I, 1991.

42. Athée-sur-Cher : 1

Bulletin de la Société Archéologique de Touraine, 33, 1963, p. 286 ; Beaumont, 1909-1910, p. LXXXVIII-XC.

43. Bossay-sur-Claise : 1

$C A G 37,4$

44. Courçay : 10

Beaumont, 1910, p. I.XXXVIII-XC; CoRdIER G., Précision sur le cimetière mérovingien du Breuil, commune de Courçay, $R A C$, XII, 1-2, 1973, p. 28-35.

45. Esvres : quantité non répertoriée

CAG 37, 121.

46. Fondettes : 768

PHILIPPON A., La trouvaille de Fondettes, BSAT, XXXI, 1956, p. 473-482.

47. Francueil : 500

Dayet, 1963, p. 240-241.

48. Langeais : 1

BOBEAU O., Le cimetière mérovingien de Langeais, BSAT, 11,1898 , p. 385-389.

49. Luzillé : 4

présence au musée de Blois en 1984.

50. Mazières-de-Touraine : 85

BOBEAU O., BSAT, 10, 2, 1892, p. 380.

51. Rochecorbon : 2

Maugard, 1981, p. 57-61.

52. Tours : 38

Theureu C., Potins des Turons au type du taureau cornupète, dits « à la tête diabolique ", BSFN, 1980, p. 692-694; FISCHER B., Le numéraire gaulois de Tours et TURONOS/TRICCOS, Cahiers Num. de la SENA, 1991, p. う̌-8. 
53. Vernou-sur-Brenne : 1

MARQUERT J.-C., Un fossé dépotoir pré-romain à Vernousur-Brenne, $R A C F, 13,1974$, p. 267-277; Maugard, 1981, p. 55-56.

LOIRE

54. Essalois : 1

PREYNAT J.-P., Anciennes et récentes découvertes numismatiques à l'oppidum d'Essalois, $R A C F, 22,1983, \mathrm{p}$. 221-238.

55. Roanne : quantité non répertoriée

BESSOU M., Études des vestiges de La Tène découverts à Roanne, Saint-Étienne, Centre d'Études Foréziennes, 1976.

\section{LOIRET}

56. Courcy-aux-Loges : 1

Nouel A., I.es découvertes des Âges du Bronze et du Fer dans le département du Loiret, Bulletin de la Société Préhistorique Française, 1957, p. 307-319.

\section{OIR-ET-CHER}

57. Couffi : quantité non répertoriée

Cothenet A., $R N, 19,1969$, p. 652.

58. Neung-sur-Beuvron : 1

ROCHE J.-L., Le théâtre gallo-romain de Neung-surBeuvron : les monnaies, RACF, 27, 1988, p. 117 et 199.

59. Pouillé : 1

Gallia, 38, 2, 1980, p. 337.

60. Vendôme : quantité non répertoriée

Blanchet, 1905 , p. $250, \mathrm{n}^{\circ} 4$.

61. Verdes : 2

$C A G 41,165$.

\section{LOZÈRE}

\section{Saint-Bonnet-de-Chirac : 1}

RICHARD J.-C., FABRIE D., Trésors et découvertes monétaires en Lozère, Cahiers Numismatiques, 1984, p. 16.

\section{MAINE-ET-LOIRE}

63. Les Alleuds : 10

Aubin G., Gruet M., Siraudeau J., Les monnaies gauloises des Pichelots, commune des Alleuds et la chronologie des potins, BSFN, 6, 1982, p. 186-188.

64. Allonnes : 3

Dei.estre X., Poplin F., Découverte d'un site de La Tène III à Allonnes, Andes, 9, 1979/1980, p. 36-42.
65. Andard : 9

CAG 49, 130.

66. Angers, la Chalouère : 5000

Aubin, 1984, p. 57-58, n².

67. Avrillé : 1

renseignement de G. Colin (inédit).

68. Beaufort-en-Vallée : 2

inventaire du musée de Beaufort-en-Vallée.

69. Beauvau : 1

renseignement de G. Colin (inédit).

70. Blou : 1

renseignement de G. Colin (inédit).

71. Brain-sur-Allonnes : 1

$C A G 49,115$.

72. Chavagnes : 1

CAG 49, 80 .

73. Chênehutte-Trèves-Cunault : 4

Gallia, 41, 1984, p. 315.

74. Guon : 1

renseignement de G. Colin (inédit).

75. Échemiré : 1

renseignement de G. Colin (inédit).

76. Le Fief-Sauvin : 35

MARTIN T., Répertoire de la Commission archéologique de l'Anjou, Angers, 1865, n²14-23, p. 11-14.

77. Mazé : quantité non répertoriée renseignement de G. Colin (inédit).

78. Montreuil-Bellay : 4

Aubin, 1984, p. 68-69, n³3.

79. Rochefort-sur-Loire : 1

CAG 49, 98 .

80. Sainte-Gemmes-sur-Loire : 1

Aubin, 1984, p. 65, n²3.

81. Saint-Georges-du-Bois : quantité non répertoriée renseignement de G. Colin (inédit).

82. Villevêque : 1

renseignement de G. Colin (inédit).

MARNE

83. La-Croix-en-Champagne : 1

présent au musée de Saint-Germain-en-Laye, classé 3452 (4515).

MAYENNE

84. Entrammes : 4

Aubin, 1981, p. 99-140.

85. Moulay : 1

Aubin, 1981, p. 99-140. 
86. Saulges : 1

Aubin, 1981, p. 99-140.

\section{MEURTHE-ET-MOSEI.I.E}

87. Saxon-Sion : quantité non répertoriée

Mitard, 1967, p. 88.

NiÈvRE

\section{Mont Beuvray : 5}

renseignements de $\mathrm{K}$. Gruel et $\mathrm{Cl}$.-A. Paratte (inédit).

89. Saint-Parize-le-Châtel : 1

SAILlANT M., Sites gallo-romains sur la commune Saint-

Parize-le-Chatel, Annales des Pays Nivernais, 1980, p. 21-23.

OISE

90. Bailleul-le-Soc : 5

Blanchet, 1905, p. 491.

91. Gournay-sur-Aronde : 5

Brunaux, Gruel, 1987.

92. Vendeuil-Caply : quantité non répertoriée

Colbert de Beaulieu, 1970, p. 110.

PARIS

93. Parvis de Notre-Dame : quantité non répertoriée

Colbert de Beaulieu, 1970, p. 109.

\section{PAS-DE-CALAIS}

\section{Béthune : 1}

présent au musée de St-Germain-en-Laye, classé 3339 (4833).

\section{PUY-DE-DÔME}

95. Corent : 2

FISCHER B., Contribution à l'étude numismatique de Corent et Gergovie, Cahiers Num. de la SENA, 1984, p. 61.

96. Gergovie : 2

Blanchet, 1905, p. 501 .

\section{SAÔNE-ET-LOIRE}

\section{Tournus : 1}

Brunaux, Gruel, 1987.

98. Chassey-le-Camp : 1

LE DANTEC J.-P., Monnaies gauloises recueillies au camp de Chassey, Cahiers Num. de la SENA, juin 1982, p. 147.

SARTHE

99. Allonnes : 222

ExDOuX H.P., Résurrection de la Gaule, Genève, 1961, chap. 9 ; Aubin, 1984, p. 34-35.

\section{SEINE-ET-MARNE}

100. Lumigny : 1

Colbert DE BEAulieu J.-B., La récolte de monnaies gauloises à Lumigny, $R B N, 1959$, p. 77.

101. Meaux : quantité non répertoriée

Colbert de Beaulieu, 1970, p. 113, note 76 .

102. Ozouer-le-Voulgis : 1

Colbert de Beaulieu, 1970, p. 109; COLBERT DE BEAULIEU J.-B., Récolte horticole de monnaies gauloises et autres bronzes à Ozouër-le-Voulgis, Cahiers Num. de la SENA, juin 1971, p. 43.

103. Saint-André-sur-Cailly : 7

SCHEERS S., Monnaies gauloises de Seine-Maritime, Rouen, 1978.

104. Sainte-Beuve-en-Rivière : 13

AubIN G., Corpus des trésors monétaires antiques de la France, $N$, Paris, 1985, p. 44.

\section{TARN}

105. Nages : 1

DEPEXROT G., AlbaGnAC I., État des découvertes et de la circulation monétaire dans la région de Montpellier, Numisma, 28, 1978, p. 241-306.

\section{VAL D'OISE}

106. Épiais-Rhus : 2

Mitard, 1967, p. 88.

\section{VAUCLUSE}

107. Cavaillon : 1

Blanchet, 1905, p. 503.

\section{VIENNE}

108. Naintré : 1

Colbert de Beaulieu, 1970, p. 109, note 49 .

109. Berrie : 1

renseignement de G. Colin (inédit).

110. Pouant : 1

renseignement de G. Colin (inédit).

111. Vézières : 1

renseignement de G. Colin (inédit).

\section{YVELINES}

112. Mézières-sur-Seine : 2

Mitard, 1967, 3, p. 87, ${ }^{\circ} 46$ et 46 bis.

113. Saint-Germain-en-Laye : quantité non répertoriée Dayet, 1963, p. 241. 
La carte de la répartition géographique montre une vaste dissémination des potins sur toute la France. Quarante départements possèdent des découvertes de ce type. Cependant, cette carte est sans aucun doute très incomplète, ceci pour plusieurs raisons : des imprécisions de dénomination dans certains ouvrages (notamment ceux datant du début du siècle) rendent difficile l'identification de ce type de pièces. On peut citer par exemple A. Blanchet qui appelle bon nombre de potins "bronze coulé au quadrupède déformé ». Comment reconnaître, lorsqu'il n'y a pas d'illustrations ni de descriptions précises, qu'il s'agit de potins «à la tête diabolique »?

De plus, ces potins souvent illisibles car trop oxydés, n'ont pas toujours été répertoriés dans les rapports de fouilles. Des archéologues méprisant ces pièces à première vue rebutantes, sans valeur ni intérêt apparent, s'abstenaient de les noter parmi leurs découvertes. C'est vraisemblablement pourquoi beaucoup de départements ne sont pas cités sur cette carte, non par l'absence de potins « à la tête diabolique », mais par manque d'informations. Au vu de cette carte, une grande quantité de potins a été découverte mais leur nombre n'a pas été répertorié. Pour ces raisons, il faut tenir compte d'un effet de contraste dû à la rareté ou au contraire à l'abondance des découvertes signalées.

\section{ATTRIBUTION}

$\mathrm{Au} \mathrm{XIX}^{\mathrm{e}} \mathrm{s}$., il semblait difficile d'attribuer à un peuple l'émission des potins «à la tête diabolique ». " Ils paraissent devoir être attribués aux Andécaves, aux Turons et aux Carnutes » (Lambert, 1844, p. 19). Puis ces pièces, appelées aussi « type de la trouvaille des Ouldes ", furent attribuées aux Turons par A. Blanchet (Blanchet, 1905). En raison de nouvelles connaissances de provenances très éloignées de la région des Turons, ce numéraire a été attribué aux Séquanes de Haute-Alsace (Forrer, 1908).

Si nous considérons les récoltes et les trésors, trois départements sont plus représentés : la Sarthe avec le sanctuaire d'Allonnes (222 potins), l'Indre-et-Loire avec des dépôts tels que celui de Fondettes (768 pièces), Francueil (500 exemplaires), Mazières-de-Touraine (85 pièces), l'oppidum d'Amboise, le Maine-et-Loire avec le trésor de la Chalouère (5000 spécimens). La très grande quantité de numéraires aux environs de Tours semble attester leur attribution au peuple des 'Turons.

\section{Chronologie}

Selon Colbert de Beaulieu, ces pièces seraient contemporaines de la guerre des Gaules. En effet, la plupart ont été découvertes dans des contextes archéologiques appartenant à la seconde moitié du $\mathrm{I}^{\mathrm{er}} \mathrm{s}$. avant J.-C. En ce qui concerne la durée d'utilisation de ces potins, elle perdure. On peut citer les découvertes faites à Tours où l'on a trouvé des potins "à la tête diabolique » dans des niveaux stratigraphiques appartenant au $\mathrm{III}^{\mathrm{e}} \mathrm{s}$. après J.-C. Cependant, il faut bien admettre que le temps de circulation des potins a été variable selon les régions.

Les potins "à la tête diabolique ", mais également l'ensemble des types de potins restent encore, par certains côtés, assez énigmatiques. Une question résume bien l'ensemble des problèmes que présentent ces objets monétiformes : nous avons affaire à des pièces sans valeur marchande (alliage de très mauvaise qualité), d'une technique très rudimentaire, ce qui permet de confirmer l'absence de contrôle d'un pouvoir émetteur. Pourtant les possesseurs de ces pièces les réunissent dans des vases clos et les enfouissent afin de les protéger, de les dissimuler : pourquoi? Les dépôts dans la région des Turons sont nombreux. Les précautions prises par les gens pour protéger ces pièces des guerres, des révoltes, de l'insécurité, prouvent que les potins possédaient une valeur certaine, une fonction particulière au sein de la société gauloise et gallo-romaine. S'ils n'avaient eu qu'une fonction de monnaie fiduciaire, il serait curieux que les personnes aient pris toutes ces mesures à leur égard. Les connaissances et l'exploitation judicieuse des futures découvertes archéologiques apporteront à plus ou moins longue échéance, les réponses à toutes ces questions.

Florence BARTHÉLÉMY

\section{Bibliographie}

AUBIN G.

1981, Aspects de la circulation monétaire en Mayenne dans l'Antiquité, Revue de la société d'archéologie et d'histoire de la Mayenne.

AUBIN G.

1984, Corpus des trésors monétaires antiques de la France, III.

BEAUMONT de

1909-1910, Sépultures de la Volandrie, Bulletin de la Société Archéologique de Touraine, 17. 
CAC 35

Carte archéologique de la Gaule, 35 Ille-et-Villaine, Paris, 1990.

\section{CAG 37}

Carte archéologique de la Gaule, 37 Indre-et-Loire, Paris, 1988.

\section{CAG 41}

Carte archéologique de la Gaule, 41 Loir-et-Cher, Paris, 1988.

CAG 49

Carte archéologique de la Gaule, 49 Maine-et-Loire, Paris, 1988.

COLbert de BEAulieu J.-B.

1961, Notices de numismatique armoricaine, Annales de Brelagne, LXVIII.

DAYET M.

1963, Trouvaille de monnaies gauloises des environs de Loches, Bulletin de la soc. française de numismatique, 4, 1963.
FISCHER B.

1986, Les collections numismatiques de la société Dunoise. Les monnaies gauloises, Bulletin de la société archéologique d'Eureet-Loir, pl. XV-XIX.

LEMIÈRE P.-L.

1852, Essai sur la numismatique gauloise du Nord-Ouest de la France, Bulletin archéologique de l'association bretonne, p. 203-235.

MAUGARD R.

1981, Monnaies en Touraine, RACF.

MITARD P.-H.

1967, Les monnaies gauloises du Vexin français, Bulletin de la société archéologique du Vexin français.

VIAN C.

1966, Trouvailles de monnaies antiques dans la région du Vaucluse, Cahiers Num. de la SENA, 3. 